Article

\title{
Automatic Detection System of Deteriorated PV Modules Using Drone with Thermal Camera
}

\author{
Chris Henry ${ }^{1}$, Sahadev Poudel ${ }^{1}$, Sang-Woong Lee ${ }^{1}\left[\right.$ and Heon Jeong ${ }^{2, *}$ \\ 1 Pattern Recognition and Machine Learning Laboratory, Gachon University, Seongnam 13120, Korea; \\ engr.chrishenry@gmail.com (C.H.); sahadevp093@gmail.com (S.P.); slee@gachon.ac.kr (S.-W.L.) \\ 2 Department of Fire Service Administration, Chodang University, Mu-An 58530, Korea \\ * Correspondence: hjeong@cdu.ac.kr; Tel.: +82-61-450-1229
}

Received: 28 April 2020; Accepted: 26 May 2020; Published: 29 May 2020

\begin{abstract}
In the last few decades, photovoltaic (PV) power station installations have surged across the globe. The output efficiency of these stations deteriorates with the passage of time due to multiple factors such as hotspots, shaded cell or module, short-circuited bypass diodes, etc. Traditionally, technicians inspect each solar panel in a PV power station using infrared thermography to ensure consistent output efficiency. With the advancement of drone technology, researchers have proposed to use drones equipped with thermal cameras for PV power station monitoring. However, most of these drone-based approaches require technicians to manually control the drone which in itself is a cumbersome task in the case of large PV power stations. To tackle this issue, this study presents an autonomous drone-based solution. The drone is mounted with both RGB (Red, Green, Blue) and thermal cameras. The proposed system can automatically detect and estimate the exact location of faulty PV modules among hundreds or thousands of PV modules in the power station. In addition, we propose an automatic drone flight path planning algorithm which eliminates the requirement of manual drone control. The system also utilizes an image processing algorithm to process RGB and thermal images for fault detection. The system was evaluated on a 1-MW solar power plant located in Suncheon, South Korea. The experimental results demonstrate the effectiveness of our solution.
\end{abstract}

Keywords: photovoltaic power station; fault detection; autonomous drone; thermal image analysis

\section{Introduction}

The development of renewable energy has gained a lot of attention worldwide. This increased interest in renewable energy is primarily due to environmental problems such as global warming, abnormal weather, depletion of fossil fuels, etc. Among various renewable energy sources, solar energy has attracted much attention in the renewable energy sector as a pollution-free, sustainable, and abundant energy source. Photovoltaic (PV) power station installations have significantly increased worldwide over the past few years. A PV power station typically consists of hundreds or thousands of PV modules which are connected together in a series circuit. These PV modules are the key components in a solar power station since the output efficiency of these stations are dependent upon these modules.

Unfortunately, PV modules are subject to various defects with the passage of time, which leads to adverse problems such as low-power efficiency. Usually, the electrical characteristics of all PV cells in the string are similar and operate at the maximum power point (MPP) current to achieve each cell's optimal output. However, when there is a discrepancy among the cell electrical characteristics, the single string current is not able to operate at every cell's MPP, which causes minimal performance [1]. The most common factors that lead to such discrepancy are partial shading, degradation, short-circuited bypass diodes, etc. When there is one low current PV cell (e.g., due to weed shadow) in the string of several high short-circuit current PV cells, the forward bias across all of these cells reverse biases 
the shaded cell, which drastically increases the cell temperature and leads to hot spotting [2]. It can damage the cell and reduce the total power output of the solar panel.

Therefore, regular inspection of PV modules is critical for maintaining an optimal output efficiency. Methods for PV module inspection include manual inspection, laser detection [3], satellite observations [4,5], infrared thermography, and electroluminescence imaging [6-8]. Manual inspection is highly time inefficient while laser detection and electroluminescence imaging cannot be applied to PV power stations. Infrared thermography gained significant attention due to its ease of use and applicability to large-scale PV systems. In infrared thermography, a technician usually inspects each PV module with a handheld infrared camera. Inspecting a single PV module is effortless; however, inspecting a large-scale PV system is a considerably lengthy procedure because large-scale PV systems consist of hundreds or thousands of PV modules. In the last few years, the academic and industrial communities are interested in time efficient drone-based infrared thermography methods. Typically, a drone equipped with thermal camera is operated wirelessly by a technician and images are captured and saved during the flight, which are later processed for fault detection. Most previous drone-based approaches [9-11] require manual drone control, which is an exhausting procedure since it involves manual labor. In addition, previous works [12-14] are unable to provide the exact locations of the defective solar panels, which further delays the recovery process.

This paper presents an autonomous drone-based infrared thermography solution for PV module fault detection and localization. The developed drone system consists of a gimbal-equipped drone based on Pixhawk 2.1 flight controller (FC). The drone was designed as a 1.1-m class hexacopter drone supporting a payload of $2 \mathrm{~kg}$ from K-Pro Systems, Seoul, Korea. A dual camera setup consisting of an RGB camera (Logitech C270 (Logitech, Suzhou, China)) and a thermal camera (FLIR Vue Pro R (FLIR Systems, Inc., Wilsonville, OR, USA)) was mounted on the drone system. Raspberry Pi 3 was used as the mainboard for interfacing with the dual camera setup and FC. The RGB camera was interfaced to Raspberry Pi via the USB port while the FC and the thermal camera were linked via MAVlink. The captured images, global positioning system (GPS) information, and drone status information are stored on an SD card connected to the mainboard. The saved information is later processed on an offline general-purpose computer. The proposed drone system can fly autonomously over an automatically planned flight path by our flight planning algorithm. As automatic hot-spot localization is one of the essential aspects of PV plant inspection, our system can estimate the exact GPS location of the faulty PV modules among thousands of PV modules. The main contributions of our study are as follows:

1. We propose an automatic drone-based solution that can operate autonomously with minimal user intervention.

2. The proposed solution can detect and exactly locate the faulty PV modules in a large-scale PV power station.

3. Our approach was tested in a real environment on a large-scale 1-MW PV power station, demonstrating the practical applicability of our work.

The remainder of the paper is organized as follows-Section 2 reviews related studies along with their limitations. The details about our hardware and software design are described in Section 3. Performance evaluation is presented in Section 4 . The conclusions are covered in the last section of this paper.

\section{Related Works}

This section discusses about previous drone-based infrared thermography methods for PV module fault detection and the limitations of those works. Researchers across the world have studied infrared thermography for more accurate solar panel fault detection. Quater et al. [12] proposed a novel monitoring system using UAV that detects several photovoltaic modules' failures. Thermal imaging and visual cameras were used as monitoring tools for inspecting PV power station. Aghaei et al. [13] 
designed an algorithm which detects the defects and failures on PV systems using thermography analysis. Their algorithm detected the hot temperature area in infrared images.

First, the original image was transformed to gray scale, which was followed by Gaussian filtering to reduce noise. Then, binary model was applied on the resulting images to distinguish hot and cold regions in the PV modules. Finally, the use of Laplacian model presented the characteristics of defective parts and determined the boundary area of the panel. A similar approach was proposed by Aghaei et al. [15], who computed the percentage of degradation and boundary area. Aghaei et al. [14] captured images using a drone at varying altitude to investigate the relationship between the altitude of image capture and possible defect identification in PV modules. However, these methods [12-14] can only detect the defects and not the exact location of the fouled PV module. This limitation hinders fast repairing process after detection of defective modules. Dotenco et al. [16] proposed image processing pipeline for the automatic detection of individual photovoltaic modules, analysis of malfunctioned modules using infrared thermography, and classification into three main groups: overheated modules, hot spots, and overheated substrings. Lee et al. [17] proposed a solar panel fault detection system using drones, thermal images, and RGB images. They detected the position of the solar panel array using RGB image and used thermal images to detect the faulty panels. Although these methods [15-17] can detect and localize the defective modules, they have been tested on a small scale dataset or in an artificial environment. On the contrary, some studies [9-11] have focused on the detection of fouling in large-sale PV panel systems. In [9], a computer vision approach was applied to identify and detect anomalies in PV modules in large-scale PV plants. The authors integrated geographic information with the results of template matching algorithm applied to thermal images, allowing panel identification by assigning identifier to each module during different flight sessions. In [10], a drone-mounted infrared thermography system was designed to rapidly detect fouling on PV panel systems. K neighbor mean filter was used for preprocessing the infrared images and single PV module in each image was recognized and extracted. In [11], the internal and external faults were detected by using thermal and charge-coupled device (CCD) cameras mounted on a drone. Morphological transformations and canny edge detection were used to find hotspots in the modules and the seed selection algorithm was used to determine the defective and the background pixels. These methods can detect and localize faulty modules and have been tested on real large-scale PV power stations. However, the drones used in these methods are remotely controlled by a technician, which adds manual labor to the procedure.

\section{Limitations}

For a fair comparison, we present the strengths and weaknesses of previous works along with the proposed method in Table 1. Most of the previous works have at least one of the following limitations:

- The drones are controlled remotely in most previous approaches [9-11], which puts a burden on the technicians. Remotely controlling a drone in large PV stations can take several minutes to completely monitor the whole area.

- Previous works [12-14] are unable to localize the exact location of the faulty PV module. The location of the defective module is crucial for quick repairing process in case of large-scale PV systems.

- Most researchers [15-17] have tested their approaches on small datasets or in artificial environments. This can limit the practical applicability of their works. 
Table 1. Comparison between proposed approach and related works in terms of strengths and weaknesses.

\begin{tabular}{|c|c|c|}
\hline Method & Strengths & Weaknesses \\
\hline Quarter et al. [12] & $\begin{array}{l}\text { - Detect several PV module's failure. } \\
\text { - Tested on large dataset: } 10 \text { polycrystalline } \\
\text { silicon modules. }\end{array}$ & $\begin{array}{l}\text { - Unable to localize faulty } \\
\text { PV modules. }\end{array}$ \\
\hline Aghaei et al. [15] & $\begin{array}{l}\text { - Compute percentage of damage and } \\
\text { degradation. } \\
\text { - Localize faulty modules. }\end{array}$ & $\begin{array}{l}\text { - Lacks testing on large-scale } \\
\text { PV power station. }\end{array}$ \\
\hline Dotenco et al. [16] & $\begin{array}{l}\text { - Detection of PV modules and classification } \\
\text { of defects. } \\
\text { - Identifies significant temperature } \\
\text { abnormalities. } \\
\text { - Localize faulty modules. }\end{array}$ & $\begin{array}{l}\text { - Remote controlled } \\
\text { - Lacks testing on large-scale } \\
\text { PV power station. }\end{array}$ \\
\hline Aghaei et al. [13] & $\begin{array}{l}\text { - Efficient real time diagnostics } \\
\text { and inspection. } \\
\text { - Compute degradation percentage and } \\
\text { boundary area. }\end{array}$ & $\begin{array}{l}\text { - Does not specify specific } \\
\text { defects. } \\
\text { - Unable to localize faulty PV } \\
\text { modules. } \\
\text { - Lacks testing on large-scale } \\
\text { PV power station. }\end{array}$ \\
\hline Aghaei et al. [14] & $\begin{array}{l}\text { - Identify the correlation between altitudes } \\
\text { of aerial images taken by the UAV and } \\
\text { possible defects' identification. }\end{array}$ & $\begin{array}{l}\text { - Remote controlled } \\
\text { - Unable to localize faulty PV } \\
\text { modules. } \\
\text { - Lacks testing on large-scale } \\
\text { PV power station. }\end{array}$ \\
\hline Addabbot et al. [9] & $\begin{array}{l}\text { - Information integration from multiple } \\
\text { sources. } \\
\text { - Panel identification and detection of } \\
\text { thermal anomalies. }\end{array}$ & $\begin{array}{l}\text { - Requires geo-referenced } \\
\text { data which are not available } \\
\text { at all times. } \\
\text { - Lacks testing on large-scale } \\
\text { PV power station. }\end{array}$ \\
\hline Zhang et al. [10] & $\begin{array}{l}\text { - Localize faulty modules. } \\
\text { - Tested on large scale datasets. }\end{array}$ & - Remote controlled \\
\hline Alsafasfeh et al. [11] & $\begin{array}{l}\text { - Real time inspection and fault detection } \\
\text { - Localize faulty modules. } \\
\text { - Tested on Large scale datasets. }\end{array}$ & - Remote controlled \\
\hline Lee et al. [17] & - Localize faulty modules. & $\begin{array}{l}\text { - Remote controlled } \\
\text { - Lacks testing on large-scale } \\
\text { PV power station. }\end{array}$ \\
\hline Proposed method & $\begin{array}{l}\text { - Localize faulty modules. } \\
\text { - Tested on Large scale datasets. } \\
\text { - Autonomous drone control. }\end{array}$ & $\begin{array}{l}\text { - Cause of detected fault must } \\
\text { be figured out manually. }\end{array}$ \\
\hline
\end{tabular}

\section{System Configuration}

The overall workflow of our system is presented in Figure 1. Our proposed solution consists of the following main steps:

1. Prepare drone's hardware structure which includes mounting of the dual camera setup.

2. Plan the flight's path automatically with flight planning algorithm and feed it to the drone.

3. The drone flies over the designated path autonomously and the captured images are saved to an SD card along with the GPS information.

4. The saved images are processed using our proposed image processing algorithm on a desktop computer.

5. The locations of the defective solar panels are displayed to the user. 


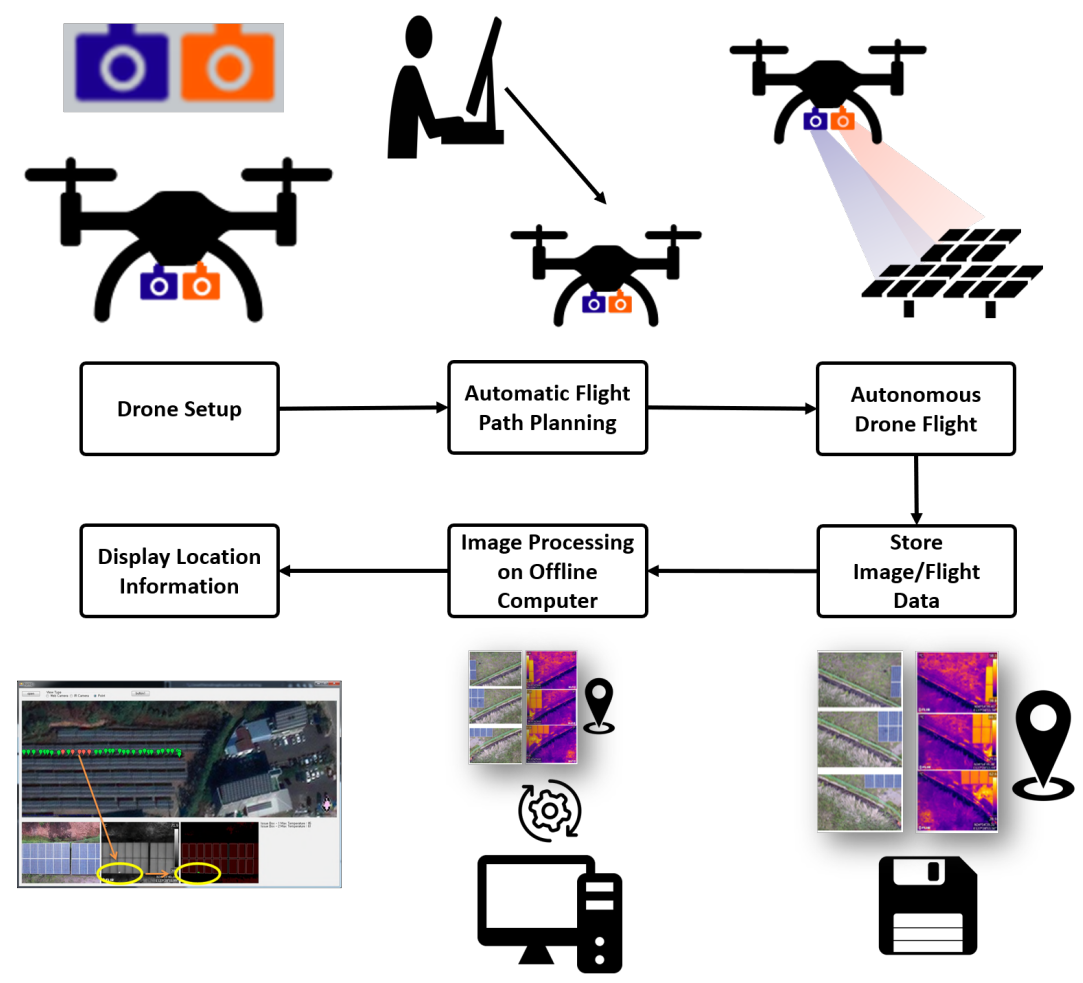

Figure 1. Block diagram of overall system.

\subsection{Drone Hardware Details}

We developed a gimbal-equipped drone based on the Pixhawk 2.1 flight controller. A dedicated ground control station (GCS) program was also developed based on C Sharp. The drone is a 1.1-m hexacopter class drone which can carry a payload of $2 \mathrm{~kg}$ and has a flight time of $20 \mathrm{~min}$. A XEON $6 \mathrm{~S} 22,000 \mathrm{mAH}$ high voltage lipo battery with twelve supporting motors was selected, having two serial connections. It holds a higher voltage charge per cell, which results in more potential power for the drone and improves the flight time efficiency by $20 \%$. A picture of the designed drone and a block diagram of our drone system are shown in Figures 2 and 3, respectively. The drone consists of the three main components:

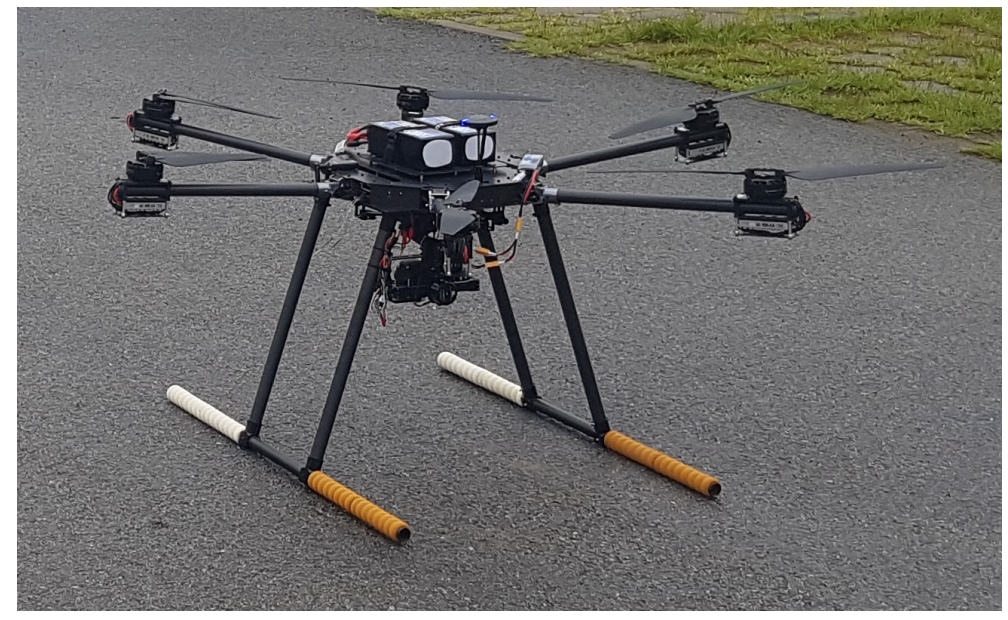

Figure 2. Physical structure of the drone. 


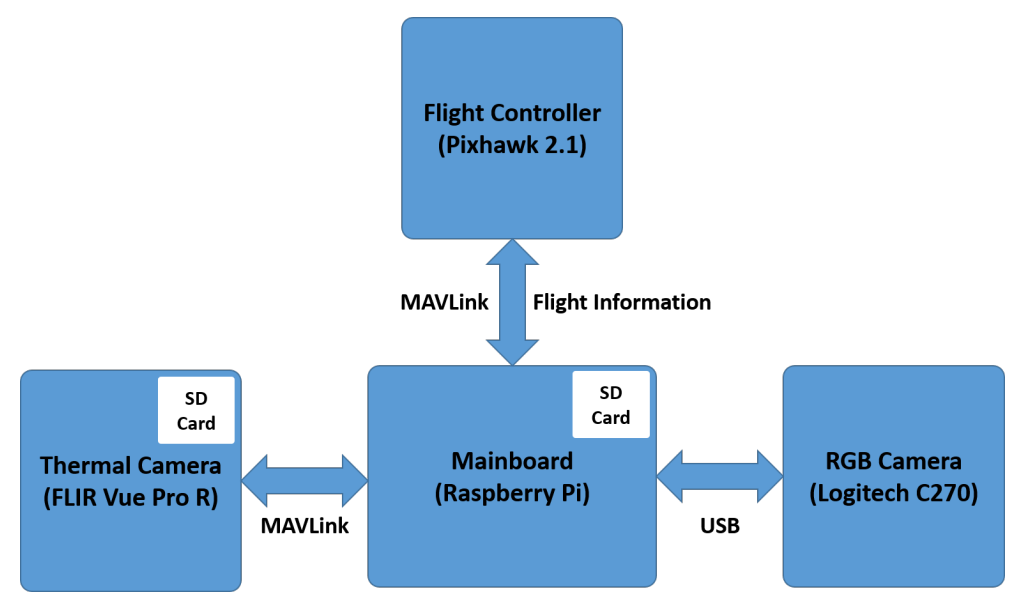

Figure 3. Block diagram of proposed drone system.

\subsubsection{Mainboard (Raspberry Pi 3)}

This study uses Raspberry Pi 3 Model B+ as the mainboard for the drone system. It is mainly responsible for capturing/saving images and for saving the flight information for later use. Raspberry $\mathrm{Pi}$ is a credit-card sized computer developed by the Raspberry Pi Foundation in the United Kingdom. Raspberry Pi 3 Model B+ is equipped with four Cortex-A53 CPUs clocked at 1.4 GHz. A total of $1 \mathrm{~GB}$ of RAM along with four USB 2.0 ports, HDMI output, LAN port, Wi-Fi, and a MicroSD card port are available onboard the Raspberry Pi 3 Model B+. Raspberry Pi was selected as the mainboard due to its compact size, low weight, and cost efficiency.

\subsubsection{Dual Camera Setup (Thermal and RGB Camera)}

The dual camera system consists of a FLIR Vue Pro R thermal camera and a Logitech C270 RGB camera, as shown in Figure 4. FLIR Vue Pro R has a sensor resolution of $336 \times 256$ and uses an uncooled VOx microbolometer as the detector. The thermal camera weighs about 92.13-113.4 g, has dimensions $5.74 \times 4.44 \mathrm{~cm}$, and operates in a spectral range of 7.5-13.5 $\mu \mathrm{m}$, which is enough for our imaging requirements. The Logitech C270 RGB is a fixed focus camera supporting a maximum resolution of $1280 \times 720$ at 30 frames per second.

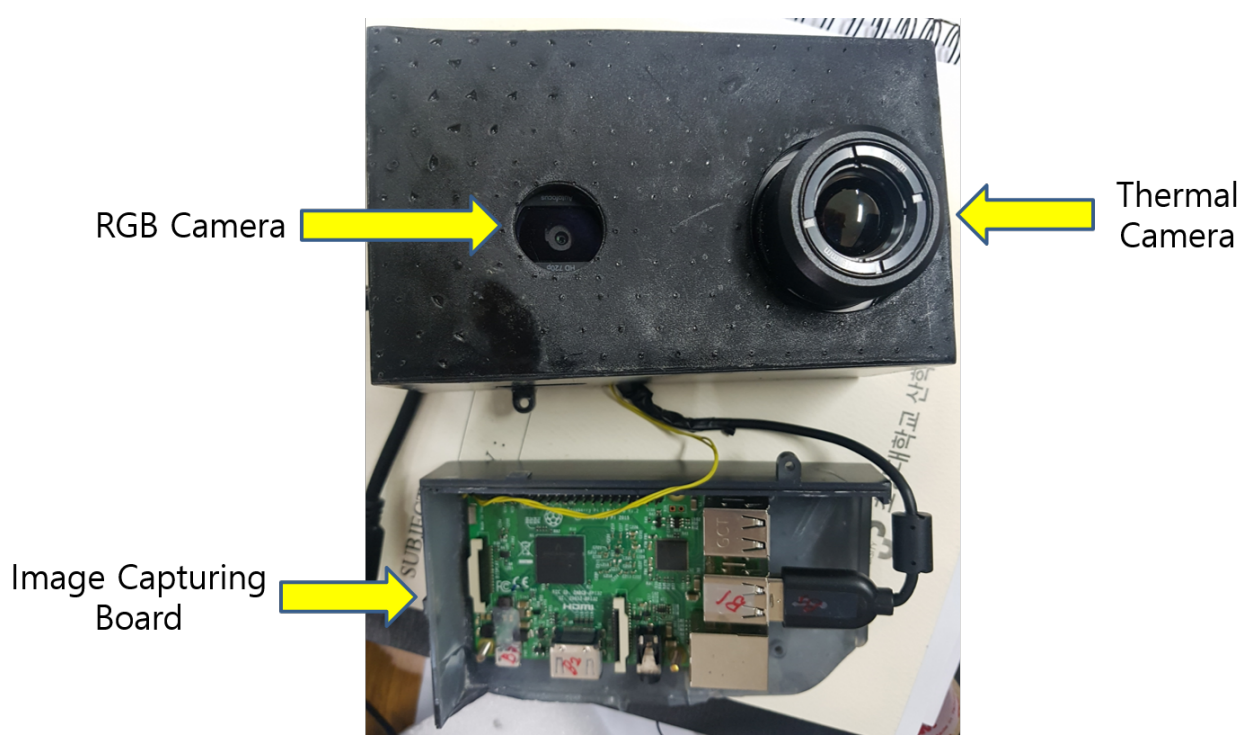

Figure 4. Physical structure of dual camera device and image capturing board. 


\subsubsection{Flight Controller (Pixhawk 2.1)}

Pixhawk 2.1 was used as the flight controller (FC) for the hexacopter drone system. Pixhawk is an open source project that has gained a lot of interest in the recent years. It is expected to secure a lot of investment and lead the fourth industrial revolution. It consists of a 32-bit ARM Cortex-M4F with floating-point unit (FPU), MPU6000 as main accelerometer and gyroscope, MEAS barometer, 5x UART, etc. The Pixhawk flight controller is more advanced than the PX4 flight control system and consists of PX4-IO and PX4-FMU controllers all integrated into a single chip along with additional IO, memory, and many other features. It supports the Unix/Linux programming environment and provides strong development capabilities by using autopilot function and multithreading. In addition, it can automatically detect and configure most peripherals. Moreover, it can support faster response, accurate positioning, and open source codes.

\subsection{Software Implementation Details}

The entire software was developed in C Sharp programming language and OpenCVSharp v3.4.1 was used as the image processing library. GMap.NET.Windows v1.8.5 was used for integrating Google Maps into the proposed system whereas Newtonsoft.Json v9.0.1 was utilized for using MAVLink. Entity Framework v6.1.3 was used for database management.

\subsection{Autonomous Flight Path Planning Algorithm}

This step is responsible for planning the flight path of our drone system. The overall block diagram of the flight planning algorithm is shown in Figure 5. An image of the whole PV power station downloaded from Google Maps. In the absence of internet service, an alternate approach for getting the image of the entire PV power station is to use image stitching. The drone is flown over the entire PV power station and images corresponding to various parts of the power station are captured. The captured images are stitched using image stitching technique, which results in an image mosaic that includes the whole power station, as shown in Figure 6b. After obtaining the image (see Figure 7a), we need to segment the solar panels from the image.

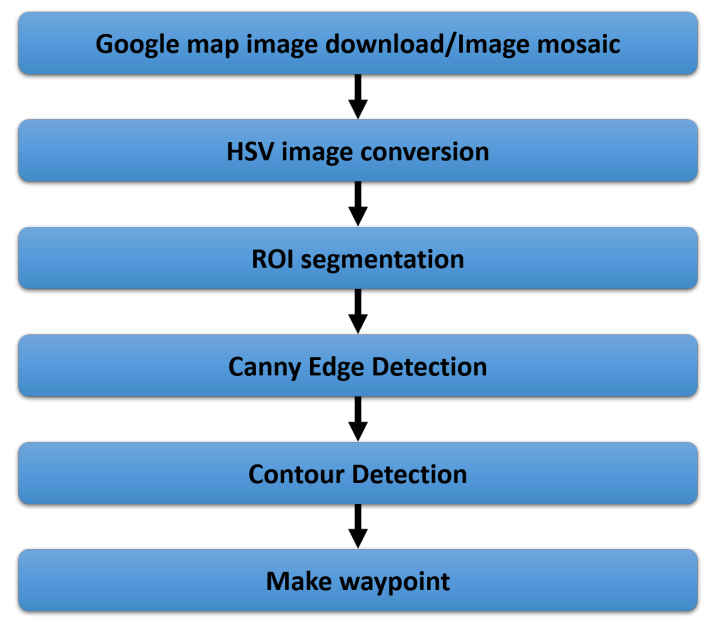

Figure 5. Block diagram of the flight planning algorithm.

Since solar panels have homogenous color, we opted for color-based segmentation. For colorbased segmentation, the image is converted into HSV color space in order to separate chroma information from intensity information. This is followed color thresholding in the HSV (Hue, Saturation, Value) color space to extract the region of interest (ROI), which in our case is the solar panel area. A threshold with a lower limit of $(80,65,65)$ and an upper limit of $(140,255,255)$ was selected based on the fact that most of the solar panels are blue in color. 

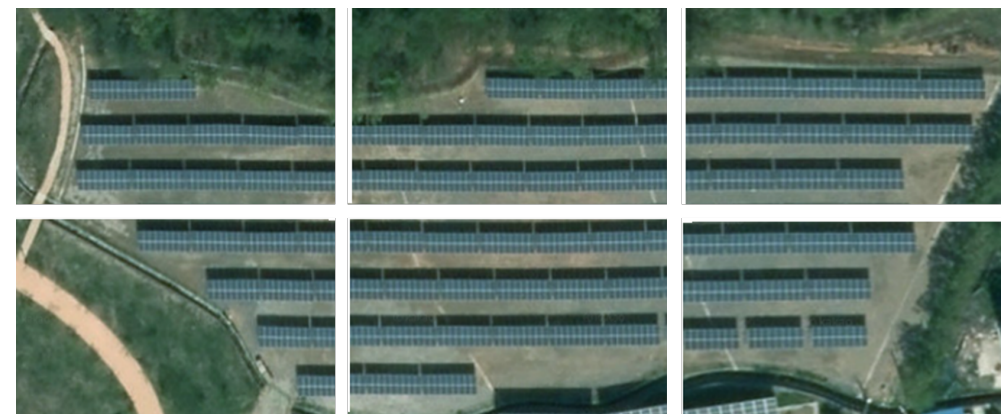

(a)

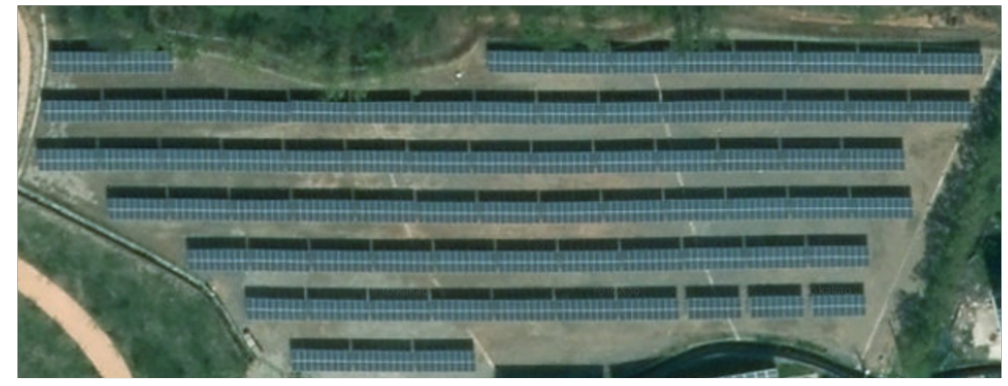

(b)

Figure 6. (a) Aerial images captured by drone; and (b) image of PV power station after image stitching.

The segmented image $I_{R O I}$ as shown in Figure $7 \mathrm{~b}$ is smoothed to remove noise before inputting it to the canny edge detection algorithm for edge detection. After edge detection, we find contours in the edge image $I_{e}$ (see Figure 7c) and filter out contours below a certain threshold area. Bounding boxes (see the red boxes in Figure 7d) are generated for each of the remaining contours. The left and right vertical lines of the bounding box are selected as the start (marked blue in Figure 7e) and end (marked red in Figure 7e) path points for each contour. The obtained waypoints are input to the drone GCS and set to perform the automatic flight path.

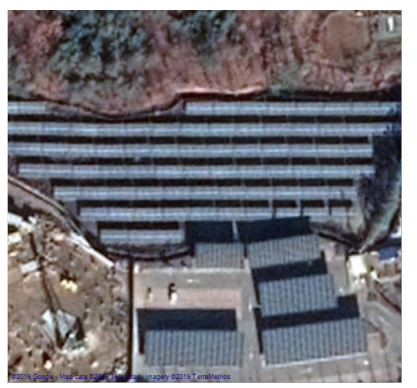

a

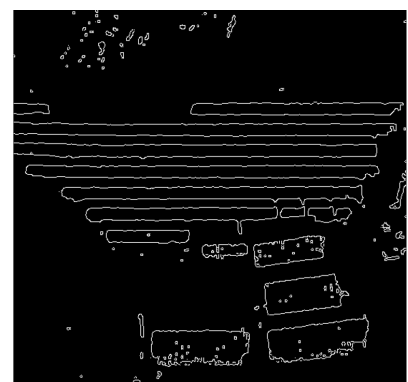

C

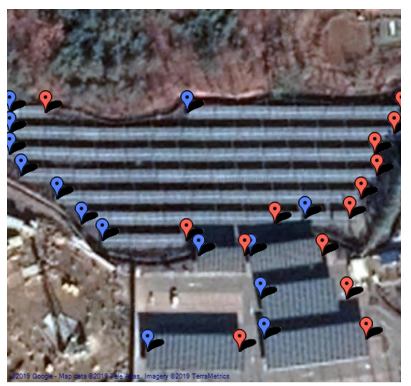

e

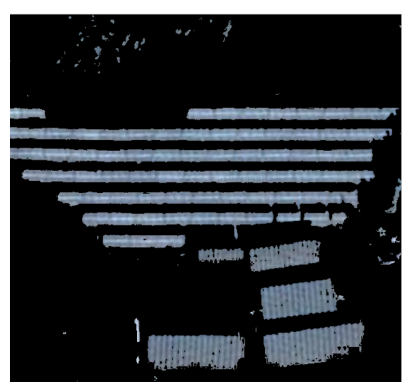

b

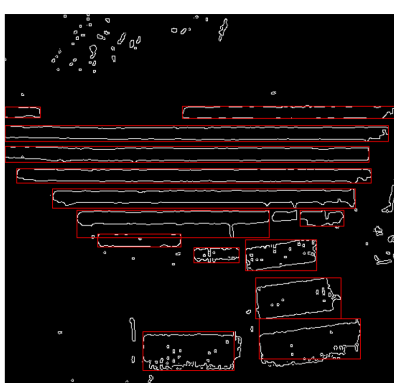

d

Figure 7. (a) Image from Google maps; (b) segmented image; (c) detected contours; (d) filtered contours; and (e) final image with markers. 
The drone captured images of the entire PV station at an altitude of $10 \mathrm{~m}$ while moving with a velocity of $3 \mathrm{~m} / \mathrm{s}$. RGB and Thermal images were captured at an interval of $1 \mathrm{~s}$ with a resolution of $1024 \times 768$ and $640 \times 480$, respectively. The captured images were stored in internal SD card. A trigger signal from the main controller was used to capture images from thermal and RGB camera simultaneously.

\subsection{Thermal Image Processing}

The proposed system uses the thermal image processing algorithm that was proposed in our previous study [18]. The overall block diagram for thermal image processing is visualized in Figure 8. This step is responsible for detecting faulty PV modules using image processing. Let $I_{R G B}$ and $I_{t h}$ denote the images captured by the RGB and the thermal camera, respectively. Each step of the image processing algorithm is summarized below:

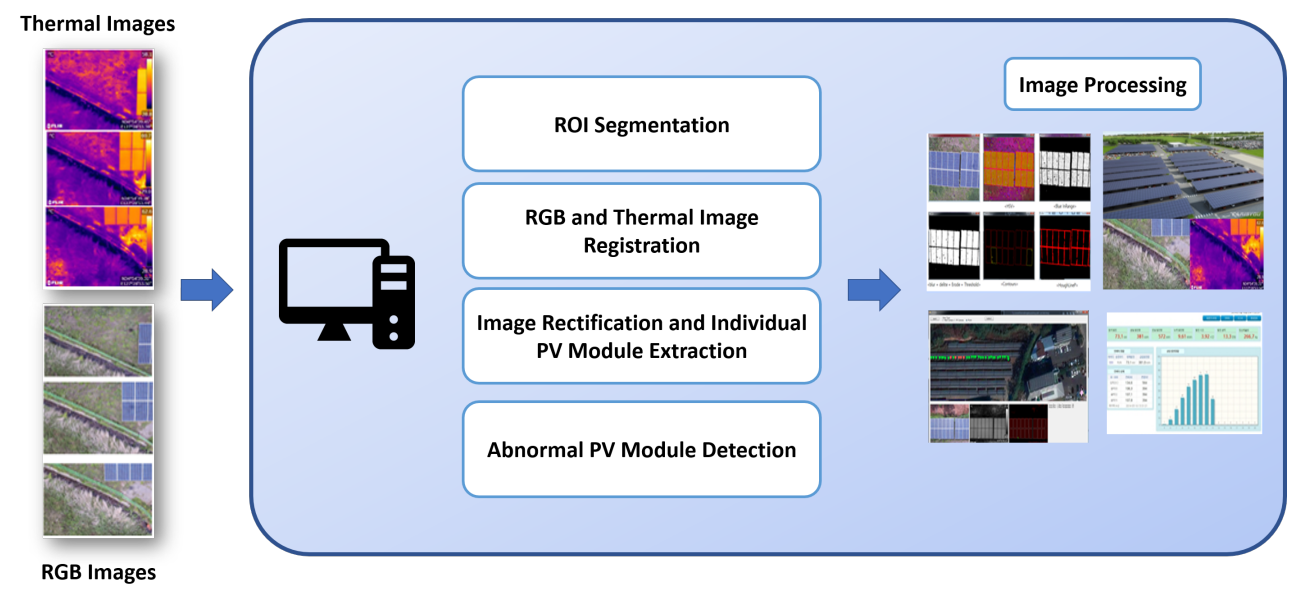

Figure 8. Block diagram for thermal image processing.

\subsubsection{ROI Segmentation}

Given image $I_{R G B}$, the PV modules are extracted from the image by using color thresholding approach similar to the one used in Section 3.2. The image is converted to HSV color space which separates the color information from the intensity information. After color space conversion, color thresholding is applied with a lower threshold limit as $(80,65,65)$ and an upper threshold limit as $(140$, $255,255)$. The resulting image $I_{R O I}$ is smoothed and inputted to canny edge detection algorithm [19]. Contours detected in the edge image $I_{e}$ are filtered based on area of the contours. Contours below a certain threshold area are removed, which results in binary image containing the borders of the PV modules. The boundaries/borders are flood filled to create a mask $M_{R G B}$ for input to the feature detection algorithm.

\subsubsection{RGB and Thermal Image Registration}

The main goal of this step is to register images $I_{R G B}$ and $I_{t h}$ since the images have a disparity/ misalignment among them. This disparity is attributed to the slight difference in the real world coordinates of both the cameras. Failure to align the images will lead to wrong localization of the faulty PV modules. The first step towards image registration is feature detection. Maximally Stable Extremal Region (MSER) [20], one of the most popular feature detectors, was used in this step of our algorithm. MSER algorithm extracts extremal regions from an image. An extremal region $R_{t}$ is essentially a connected component that is stable across multiple gray-level sets in an image. Simply, MSERs are regions which remain unchanged over a wide range of thresholds. Given an input image, MSER thresholds an image into multiple thresholded images $I_{t}$ where $t$ indicates the threshold value. The first image in the sequence would be totally black and the following images would contain white 
blobs which continue to grow until all of them merge into a totally white image. The stability of these blobs are monitored by MSER algorithm across the image sequence with respect to a function. Generally, stability of a region is defined as follows:

$$
\psi\left(R_{t}\right)=\frac{A\left(R_{t}\right)}{\frac{d}{d t} A\left(R_{t}\right)}
$$

where $A\left(R_{t}\right)$ represents the area of the region $R_{t}$ and $\frac{d}{d t} A\left(R_{t}\right)$ represents the rate of change of area. A region is stable only if its area changes slightly at different threshold values. Additionally, a stable region might be rejected if its area is below a minimum area threshold (2000 in our experiments) or above a maximum area threshold (400,000 in our experiments) value. MSER features $F_{R G B}$ and $F_{t h}$ are extracted from the RGB image $I_{R G B}$ and the thermal image $I_{t h}$, respectively. The extracted features must be matched in order to apply homography for aligning the images. For robust matching of features, the following criterion is applied:

$$
\begin{gathered}
x_{R G B}^{i} \in F_{R G B}, x_{t h}^{j} \in F_{t h}, \quad i: 1 \sim F_{\text {size }}, \quad j: 1 \sim F_{\text {size }} \\
r=\sqrt{\left(x_{R G B}^{i}-x_{t h}^{j}\right)^{2}+\left(y_{R G B}^{i}-y_{t h}^{j}\right)^{2}} \\
f(x)=\left\{\begin{array}{cc}
M_{R G B}^{k}=F_{R G B}^{i} \text { and } M_{t h}^{k}=F_{R G B}^{j}, & \text { if } r<t h_{r} \\
\text { invalid match, } & \text { otherwise }
\end{array}\right.
\end{gathered}
$$

where $\left(x_{R G B}^{i}, y_{R G B}^{i}\right)$ and $\left(x_{t h}^{j}, y_{t h}^{j}\right)$ represent the $x$ and $y$ value of the $i$ th feature point of the $F_{R G B}$ and the $j$ th element of the $F_{t h}$, respectively. $M_{R G B}$ and $M_{t h}$ are the set of valid feature points in $I_{R G B}$ and $I_{t h}$, respectively. A feature point set is only valid if the distance value $r$ between the two points is lower than the threshold distance $t h_{r}$. After matching the features, homography is applied to align the two images. Homography is the mapping between two projection planes sharing the same center of projection. It is represented as a $3 \times 3$ matrix that maps points in source image to the corresponding points in the target image. The homography matrix can be estimated by approaches such as direct linear transform (DLT) and is represented as follows:

$$
H=\left[\begin{array}{lll}
h_{00} & h_{01} & h_{02} \\
h_{10} & h_{11} & h_{12} \\
h_{20} & h_{21} & h_{22}
\end{array}\right]
$$

Let $M_{R G B}=\left\{\left(x_{R G B}^{1}, y_{R G B}^{1}\right),\left(x_{R G B}^{2}, y_{R G B}^{2}\right), \ldots,\left(x_{R G B}^{n}, y_{R G B}^{n}\right)\right\}$ be the set of matched feature points in $M_{R G B}$ and $M_{t h}=\left\{\left(x_{t h}^{1}, y_{t h}^{1}\right),\left(x_{t h}^{2}, y_{t h}^{2}\right), \ldots,\left(x_{t h}^{n}, y_{t h}^{n}\right)\right.$ be the set of corresponding feature points in $I_{t h}$. The homography matrix H maps a set of corresponding points $\left(x_{R G B}^{n}, y_{R G B}^{n}\right)$ and $\left(x_{t h}^{n}, y_{t h}^{n}\right)$ in homogeneous form as follows:

$$
\left[\begin{array}{c}
x_{R G B}^{n} \\
y_{R G B}^{n} \\
1
\end{array}\right]=H\left[\begin{array}{c}
x_{t h}^{n} \\
y_{t h}^{n} \\
1
\end{array}\right]
$$

After estimating the homography matrix, we get the registered image $I_{\text {reg. }}$. An example of the rectified image can be seen in Figure 9. 

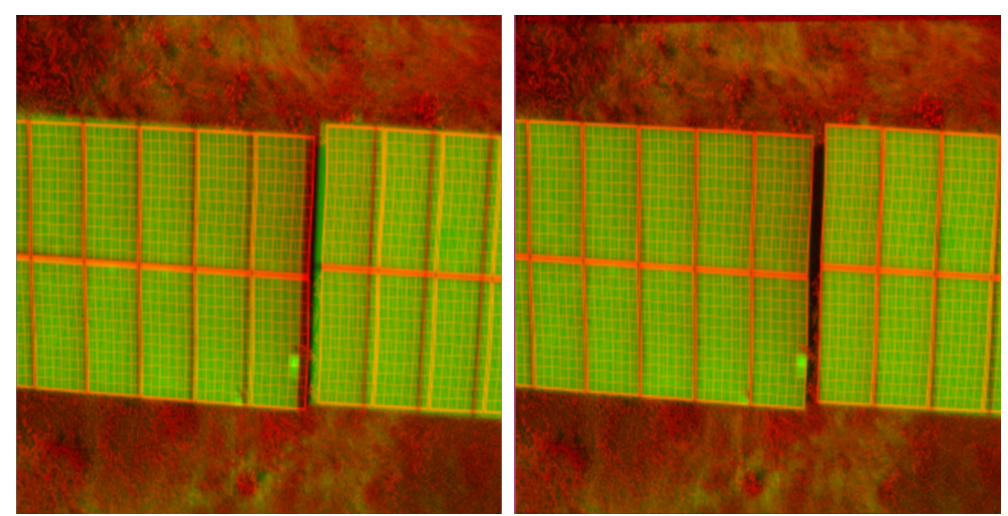

Figure 9. (left) Image before registration; and (right) image after registration.

\subsubsection{Image Rectification and Individual PV Module Extraction}

The resulting image $I_{\text {reg }}$ from previous section is rectified for easier thermal distribution analysis. A sample illustration of image rectification is shown in Figure 10a. After image rectification, the individual PV modules are extracted by using the mask $M_{R G B}$ from Section 3.4.1. Particularly, the edges of the valid feature points $M_{R G B}$ and $M_{t h}$ are used to extract the individual PV module area. An example of one of the extracted PV modules can be visualized in Figure 10b.

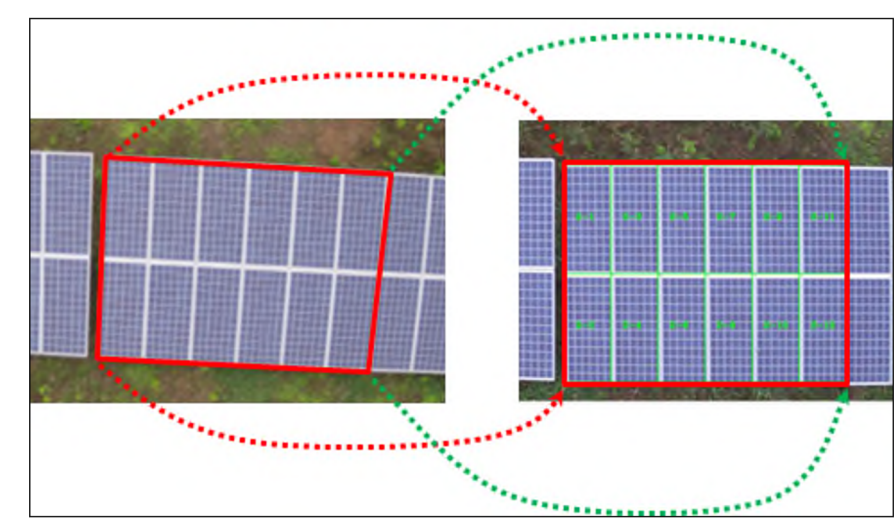

(a)

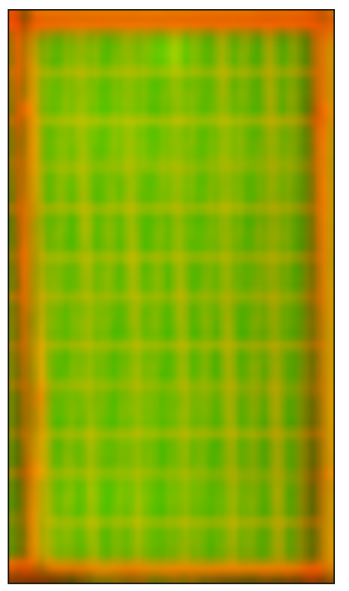

(b)

Figure 10. (a) Example of image rectification; and (b) rxample of extracted individual PV module.

\subsubsection{Abnormal PV Module Detection}

This step is responsible for classifying a PV module as defective or as normal. The decision is made based on the criterion in Equation (10). Firstly, the maximum temperature value $T_{\max }$, minimum temperature value $T_{\min }$, and average temperature value $T_{\text {avg }}$ are calculated from the thermal image. This is followed by calculating the high temperature threshold value $T_{\text {high }}$ or the low temperature threshold value $T_{\text {low }}$ using Equations (7) and (8), respectively. The count value $a_{\text {count }}$ is increased if the temperature value is lower than $T_{\max }$ and $T_{\min }$. If $a_{\text {count }}$ exceeds $0.2 \%$ of the module area value area $_{\text {module }}$, then the module is considered as abnormal.

$$
\begin{aligned}
& T_{\text {high }}=T_{\text {avg }}+T_{\max } \times 0.2 \\
& T_{\text {low }}=T_{\text {avg }}+T_{\text {min }} \times 0.2
\end{aligned}
$$




$$
\begin{gathered}
f(x)=\left\{\begin{aligned}
a_{\text {count }}++, \text { if } T_{\text {val }}<T_{\text {max }} \text { or } T_{\text {val }}<T_{\text {min }} \\
a_{\text {count }} \text { otherwise }
\end{aligned}\right. \\
g(x)=\left\{\begin{array}{cc}
\text { abnormal } P V \text { module, } & \text { if } a_{\text {count }}>\text { area } \text { arodule }_{\text {mal }} \times 0.002 \\
\text { normal } P V \text { module, } & \text { otherwise }
\end{array}\right.
\end{gathered}
$$

\section{Experimental Results, and Discussion}

To verify the validity of the developed system, photovoltaic modules were photographed by autonomous flight on a 1-MW solar power plant located in Suncheon, Korea using the proposed drone system. The flight path for the drone system was calculated by our automatic flight path planning algorithm, as explained in Section 3.2. The images captured by the dual camera device mounted on the drone were sent to the ground station. The images were then analyzed by the image processing algorithm, as explained in Section 3.3, to identify and localize defective PV modules. Figure 11a displays an image of overall PV plant, while Figure $11 \mathrm{~b}$ displays the automatically planned flight path calculated by our path planning algorithm. The drone acquired 400 thermal and visible images, which were processed by the image processing algorithm, obtaining the result displayed in Figure 11c. The various steps proposed in the algorithm contributes in distinguishing defective modules from normal modules in the PV array.

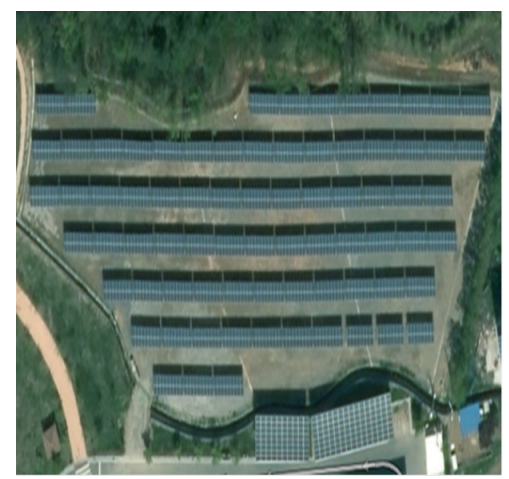

(a)

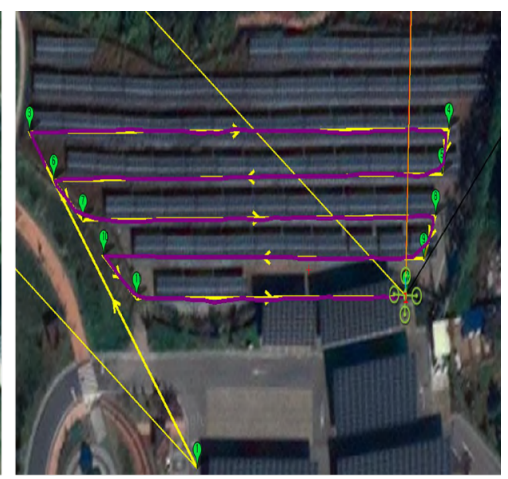

(b)

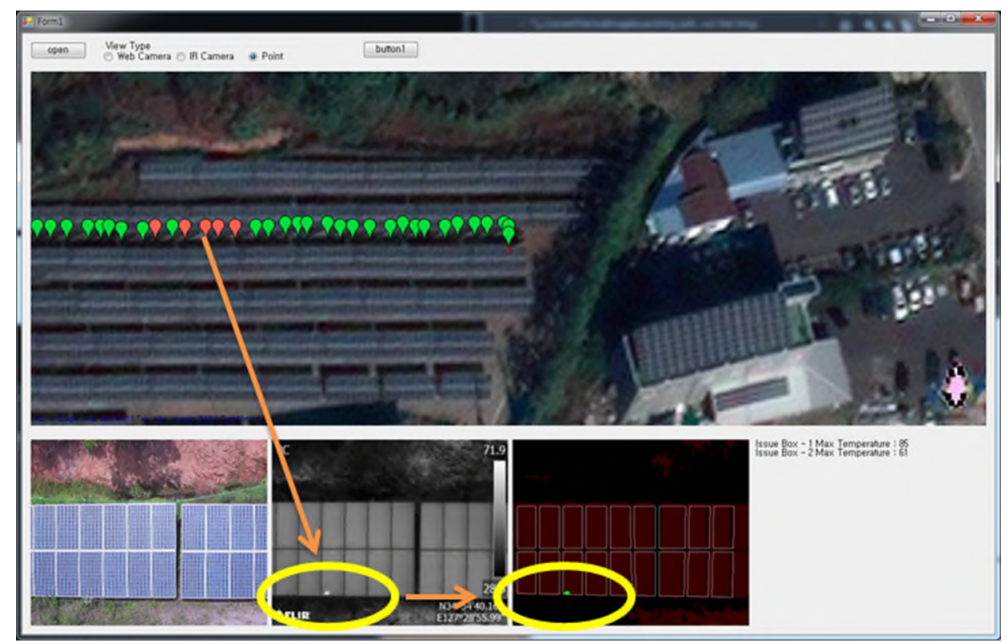

(c)

Figure 11. (a) Image of overall PV power plant; (b) image showing the automatically planned flight path; and (c) the final result showing the location of defective PV modules. 
Through image analysis, we deduced that the detection and identification depend on various characteristics of the defects such as its shape, size, location, etc. Within a photovoltaic module, a wide range of defects can occur. Many of these defects cause overheating of the entire module, single substrings, small solar cells, or even tiny cell parts. To verify the efficiency of the proposed system, three experiments were conducted, as shown in Table 2. In the first experiment, 14 defective panels were detected in different areas of the PV plant indicating the possible failures. Depending on the shape and location, the hot spots and hot areas highlighted different faults. Out of 14 defected modules, 10 were due to shade, 2 were due to the soiling matter, and the remaining 2 were due to unknown reason. However, due to PV panel reflectance by sunlight, there were 60 unknown reasons, which were caused by panel emissivity. Usually, emissivity is at its highest when the camera is perpendicular to the modules being inspected and decreases with an increasing angle [21]. To prevent these errors, we controlled the camera angle, which resulted in the second and third experiments. From the second experiment, it is observable that, out of 21 defective panels, 17 were affected by the shadow, 3 were affected by solid matter, and 1 was unknown. Figures 12 and 13 illustrate PV modules affected by shadow of weed. Among the total number of hot spots detected, the weed shadows caused the maximum number of faults. Similarly, Figure 14 shows a PV module affected by reflection due to sunlight. When using a thermal imaging camera, it is confirmed that an error may occur in absolute temperature measurement. However, it can vary with the location of panel and the angle of view taken from the camera with respect to the altitude.

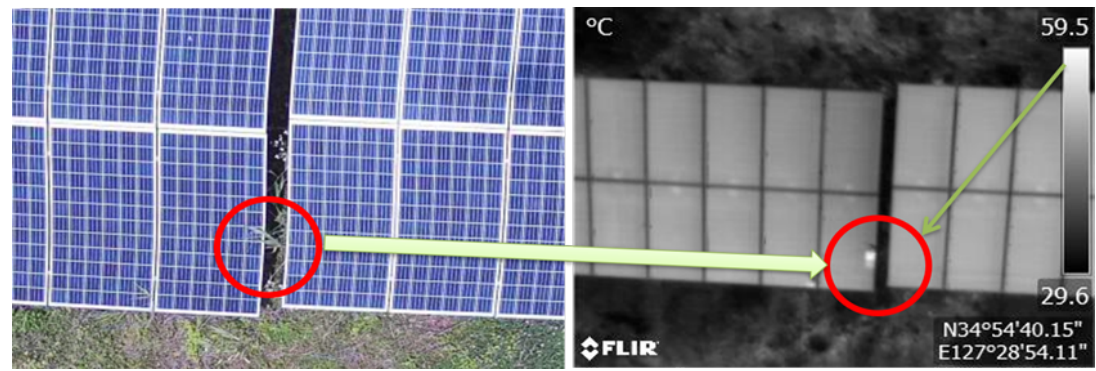

Figure 12. Hotspots caused by weed shadows: $59.5^{\circ} \mathrm{C}$.

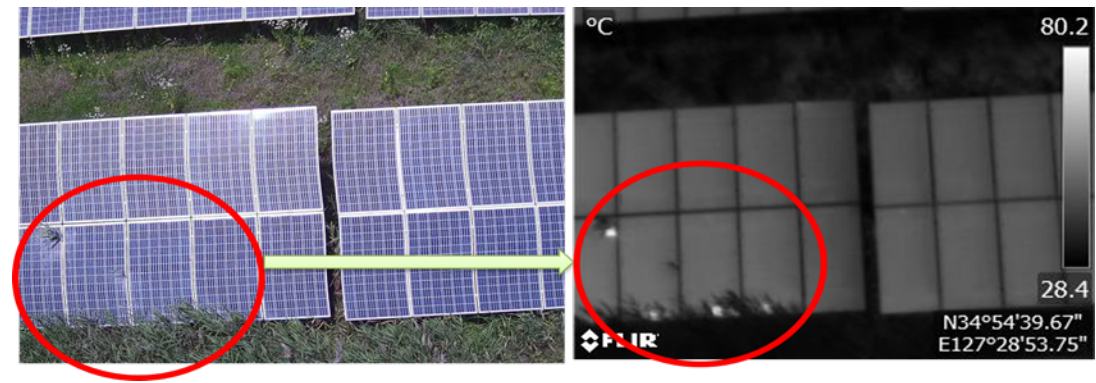

Figure 13. Hotspots caused by weed shadows: $80.2^{\circ} \mathrm{C}$.

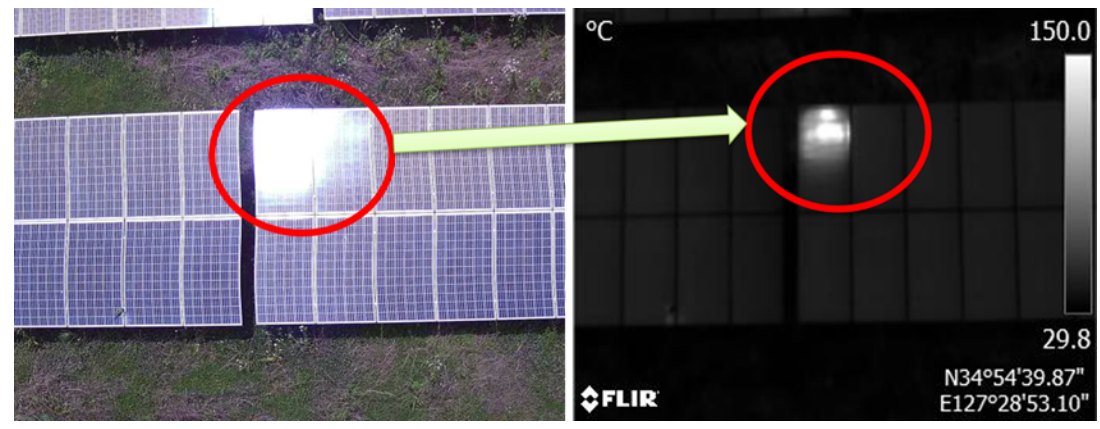

Figure 14. Error caused by sunlight reflection: $150.0^{\circ} \mathrm{C}$. 
Figure 15 shows the region where the system detected a hot spot, but the reason was unidentifiable. Different types of defects or failures can be detected only at different heights. Moreover, characteristics of defects also play an important role in its detection. Therefore, further investigation is needed to perform more efficient identification of faults. Figure 16 shows another defective PV module, which was affected by various causes such as reflection, solid, and shadow. In addition, the exact geolocalization of deteriorated PV modules in large-scale plants is one of the most critical issues in PV plant inspection. In this work, we used GPS information to locate the faulty modules. Aluminum marks can be used for thermal images georeferencing due to its low emissivity nature, which facilitates in better pointing. However, for the precise location in large-scale plants with centimeter accuracy, a further survey on RTX modules needs to be performed. In conclusion, our experiments demonstrated that hot spots generally occur due to partial shading from weeds, solid things present on the surface of PV module, broken interconnects, and/or reflection from sunlight.

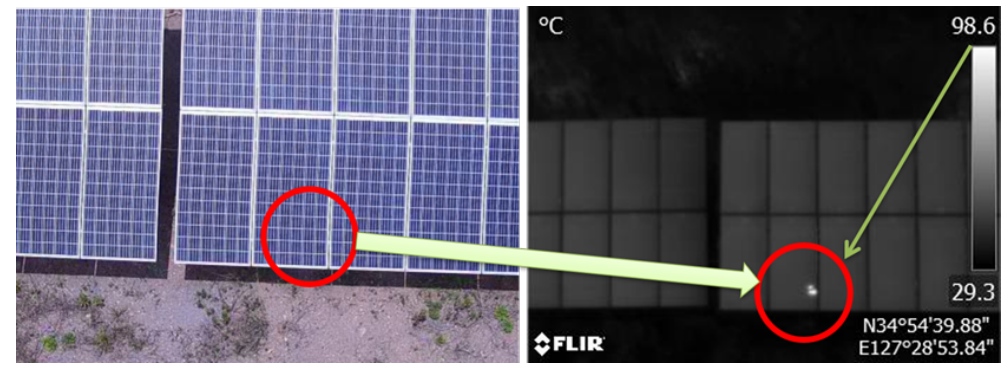

Figure 15. Hotspots caused by unknown reason: $98.6{ }^{\circ} \mathrm{C}$.
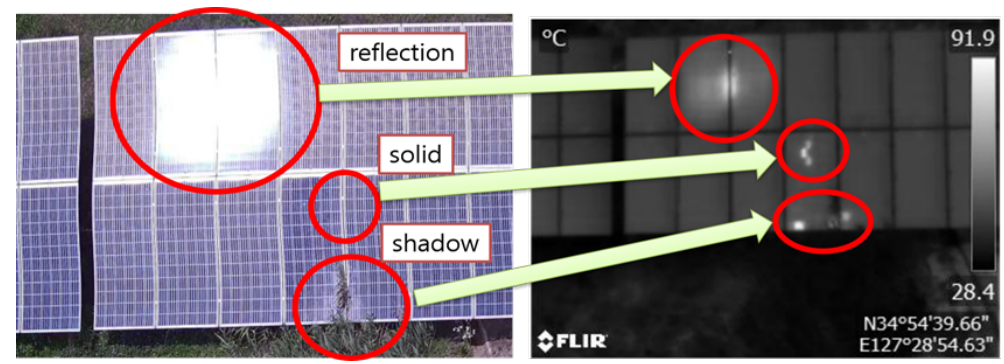

Figure 16. Hot spots caused reflection due to sunlight, solid on PV surface, and shadows.

Table 2. Results of hotspot detection and recognition.

\begin{tabular}{cccccccc}
\hline & Condition & No. of Frames & N/A (Sunlight) & Shadow & Pollution & Unknown & Total \\
\hline 1 & No control & 400 & 60 & 10 & 2 & 2 & 14 \\
\hline 2 & Angle control & 400 & 0 & 17 & 3 & 1 & 21 \\
\hline 3 & Angle control & 400 & 0 & 1 & 0 & 1 & 2 \\
\hline
\end{tabular}

\section{Conclusions}

In this paper, an autonomous drone-based infrared thermography system is proposed for automatic detection and localization of defective PV modules in a PV power station. The drone system is mounted with a dual camera setup consisting of a thermal camera and an RGB camera. The proposed drone system can fly autonomously without the need of manual control by using our automatic flight path planning algorithm. In addition, our system can detect and precisely locate the exact location of the faulty PV modules. Contrary to most previous methods, we tested our approach on a real 1-MW power plant location in Suncheon, South Korea. Our results demonstrate that the proposed method can accurately detect and locate faulty modules autonomously. 
Author Contributions: Conceptualization, H.J.; methodology, S.-W.L. and H.J.; software, C.H. and H.J.; validation, S.P., H.J. and S.-W.L.; formal analysis, S.-W.L.; investigation, C.H.; resources, S.-W.L.; data curation, H.J.; writing-original draft preparation, C.H. and S.P.; writing-review and editing, C.H. and S.P.; visualization, S.P.; supervision, H.J. and S.-W.L.; project administration, H.J.; funding acquisition, H.J. All authors have read and agreed to the published version of the manuscript.

Funding: This work was supported by the National Research Foundation of Korea (NRF) grant funded by the Korea government (MSIT) (2019R1F1A1062075).

Conflicts of Interest: The authors declare no conflict of interest.

\section{References}

1. Kaushika, N.; Rai, A.K. An investigation of mismatch losses in solar photovoltaic cell networks. Energy 2007, 32, 755-759. [CrossRef]

2. Alonso-Garcia, M.; Ruiz, J.; Chenlo, F. Experimental study of mismatch and shading effects in the I-V characteristic of a photovoltaic module. Sol. Energy Mater. Sol. Cells 2006, 90, 329-340. [CrossRef]

3. Demant, M.; Rein, S.; Krisch, J.; Schoenfelder, S.; Fischer, C.; Bartsch, S.; Preu, R. Detection and analysis of micro-cracks in multi-crystalline silicon wafers during solar cell production. In Proceedings of the 2011 37th IEEE Photovoltaic Specialists Conference, Seattle, WA, USA, 19-24 June 2011; pp. 001641-001646.

4. Drews, A.; De Keizer, A.; Beyer, H.G.; Lorenz, E.; Betcke, J.; Van Sark, W.; Heydenreich, W.; Wiemken, E.; Stettler, S.; Toggweiler, P.; et al. Monitoring and remote failure detection of grid-connected PV systems based on satellite observations. Sol. Energy 2007, 81, 548-564. [CrossRef]

5. Muselli, M.; Notton, G.; Canaletti, J.; Louche, A. Utilization of meteosat satellite-derived radiation data for integration of autonomous photovoltaic solar energy systems in remote areas. Energy Convers. Manag. 1998, 39, 1-19. [CrossRef]

6. Tseng, D.C.; Liu, Y.S.; Chou, C.M. Automatic finger interruption detection in electroluminescence images of multicrystalline solar cells. Math. Probl. Eng. 2015, 2015, 879675. [CrossRef]

7. Tsai, D.M.; Li, G.N.; Li, W.C.; Chiu, W.Y. Defect detection in multi-crystal solar cells using clustering with uniformity measures. Adv. Eng. Inform. 2015, 29, 419-430. [CrossRef]

8. Wang, C.; Jiang, X.Y.; Liu, X.H. Defect detection in crystalline silicon solar cells based on electroluminescence imaging. J. Optoelectron. Laser 2011, 9. Available online: http://en.cnki.com.cn/Article_en/CJFDTotalGDZJ201109014.htm (accessed on 28 April 2020).

9. Addabbo, P.; Angrisano, A.; Bernardi, M.L.; Gagliarde, G.; Mennella, A.; Nisi, M.; Ullo, S. A UAV infrared measurement approach for defect detection in photovoltaic plants. In Proceedings of the 2017 IEEE International Workshop on Metrology for AeroSpace (MetroAeroSpace), Padua, Italy, 21-23 June 2017; pp. 345-350.

10. Zhang, P.; Zhang, L.; Wu, T.; Zhang, H.; Sun, X. Detection and location of fouling on photovoltaic panels using a drone-mounted infrared thermography system. J. Appl. Remote Sens. 2017, 11, 016026. [CrossRef]

11. Alsafasfeh, M.; Abdel-Qader, I.; Bazuin, B.; Alsafasfeh, Q.; Su, W. Unsupervised fault detection and analysis for large photovoltaic systems using drones and machine vision. Energies 2018, 11, 2252. [CrossRef]

12. Quater, P.B.; Grimaccia, F.; Leva, S.; Mussetta, M.; Aghaei, M. Light Unmanned Aerial Vehicles (UAVs) for cooperative inspection of PV plants. IEEE J. Photovolt. 2014, 4, 1107-1113. [CrossRef]

13. Aghaei, M.; Gandelli, A.; Grimaccia, F.; Leva, S.; Zich, R. IR real-time analyses for PV system monitoring by digital image processing techniques. In Proceedings of the 2015 International Conference on Event-Based Control, Communication, and Signal Processing (EBCCSP), Krakow, Poland, 17-19 June 2015; pp. 1-6.

14. Aghaei, M.; Dolara, A.; Leva, S.; Grimaccia, F. Image resolution and defects detection in PV inspection by unmanned technologies. In Proceedings of the 2016 IEEE Power and Energy Society General Meeting (PESGM), Boston, MA, USA, 17-21 July 2016; pp. 1-5.

15. Aghaei, M.; Grimaccia, F.; Gonano, C.A.; Leva, S. Innovative automated control system for PV fields inspection and remote control. IEEE Trans. Ind. Electron. 2015, 62, 7287-7296. [CrossRef]

16. Dotenco, S.; Dalsass, M.; Winkler, L.; Würzner, T.; Brabec, C.; Maier, A.; Gallwitz, F. Automatic detection and analysis of photovoltaic modules in aerial infrared imagery. In Proceedings of the 2016 IEEE Winter Conference on Applications of Computer Vision (WACV), Lake Placid, NY, USA, 7-10 March 2016; pp. 1-9. 
17. Lee, S.; An, K.E.; Jeon, B.D.; Cho, K.Y.; Lee, S.J.; Seo, D. Detecting faulty solar panels based on thermal image processing. In Proceedings of the 2018 IEEE International Conference on Consumer Electronics (ICCE), Las Vegas, NV, USA, 12-14 January 2018; pp. 1-2.

18. Jeong, H.; Kwon, G.R.; Lee, S.W. Deterioration diagnosis of solar module using thermal and visible image processing. Energies 2020, accepted.

19. Canny, J. A Computational Approach to Edge Detection. IEEE Trans. Pattern Anal. Mach. Intell. 1986, 6, 679-698. [CrossRef]

20. Donoser, M.; Bischof, H. Efficient maximally stable extremal region (MSER) tracking. In Proceedings of the 2006 IEEE Computer Society Conference on Computer Vision and Pattern Recognition (CVPR'06), New York, NY, USA, 17-22 June 2006; Volume 1, pp. 553-560.

21. Guide to the Use of Thermal Imaging Cameras in the Construction Industry and When Working with Renewable Energy Sources. Available online: http:/ / www.flirmedia.com/MMC/THG/Brochures/T820325/ T820325_EN.pdf (accessed on 1 May 2020 ).

(C) 2020 by the authors. Licensee MDPI, Basel, Switzerland. This article is an open access article distributed under the terms and conditions of the Creative Commons Attribution (CC BY) license (http://creativecommons.org/licenses/by/4.0/). 